\title{
Generalized van der Waals theory of liquid-liquid phase transitions
}

\author{
Yu. D. Fomin, V. N. Ryzhov, and E. E. Tareyeva \\ Institute for High Pressure Physics, Russian Academy of Sciences, Troitsk 142190, Moscow Region, Russia
}

(Dated: October 1, 2018)

\begin{abstract}
In the framework of the thermodynamic perturbation theory for fluids we study how the phase diagram of an isotropic repulsive soft-core attractive potential, where a liquid-liquid phase transition exists in addition to the standard gas-liquid phase transition, changes by varying the parameters of the potential. We show that existence of the liquid-liquid transition is determined by the interplay of the parameters of the potential and the structure of a reference liquid.
\end{abstract}

PACS numbers: 61.20.Gy, 61.20.Ne, 61.20.Mv, 64.60.Kw

Despite the growing interest to the possible polymorphic phase transitions in liquids and glasses [1, 2] the nature of different phases which can be found in dense (and possibly metastable) liquids is still puzzling. The coexistence of different phases, while common for mixtures, is unexpected for a simple fluid. In principle, the rules of thermodynamics do not forbid the existence of more than two different fluid phases in a simple fluid, however, from a common experience only two of them are well known: a low density fluid (vapor) and a high density fluid (liquid) phases. At the same time in recent years experimental evidences of such features of phase diagram as liqiud-liquid transitions, polyamorphism, etc appeared for a wide range of systems including water, $S i, I, S e, S, P$, etc [1, 2, 3, 4, 5, 6, 7, 8, 9, 10, 11]. The complexity of the phase diagrams in these substances may be a result of complex interactions depending on the intermolecular orientations.

At the same time exploring the possibility that simple fluids interacting through isotropic potentials may exhibit the similar behavior represents a serious challenge for theorists.

It was shown recently through molecular dynamics simulations that a system of particles with the isotropic repulsive soft-core attractive potential may have highdensity and low-density liquid phases [19, 20]. This potential may be considered as an effective potential resulting from an average over the angular degrees of freedom for systems where the position of the minimum approach between particles depends on their relative orientations like in the case of the hydrogen bonding between water molecules [1, 5, 13, 23. . This potential may be also used to model interactions in a variety of systems including liquid metals, colloids, silica 1, 23].

After the pioneering work by Hemmer and Stell 16], where the soft core potential with an attractive interaction at large distances was first proposed for the qualitative explanation of the solid-solid critical point in materials such as Ce or Cs, a lot of attention was paid to the investigation of the properties of the systems with the potentials that have a region of negative curvature in their repulsive core. In spite of the simplicity of the model, the physical mechanism that causes the liquidliquid phase transition in such systems is not completely understood. As was emphasized in [24, 25] it arises from

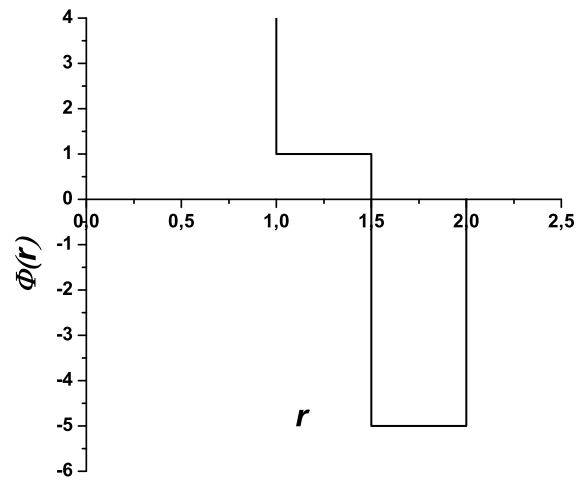

FIG. 1: An isotropic potential with an attractive well and a repulsive component consisting of a hard core plus a finite shoulder.

an interplay of the different components of the pair interaction. In Refs. 24, 25] authors tried to disentangle the role of each component to investigate the dependence of the phase diagram on the potential parameters. In Ref. 24] the results of molecular dynamics calculations performed for several sets of parameters were presented. The resulting behavior of the critical points was interpreted through a modified van der Waals equation where the effect of the repulsive shoulder at different densities $\rho$ and temperatures $T$ can be taken into account by an effective excluded volume depending on both $\rho$ and $T$. In Ref. 25] the same analysis was undertaken by using an integral equation approach in the hypernetted-chain approximation. In Refs. 24, 25] it was shown that the high-density liquid-liquid critical point can be found only when there is some kind of balance between the attractive and repulsive parts of the potential.

It should be noted that it is widely believed (see, for example, [26, 27] ) that a fluid-fluid transition should be related to the attractive part of the potential. However, in Ref. 28 in the framework of the generalized van der Waals theory it was shown that the purely repulsive step potential is sufficient to explain the high density liquidliquid phase transition. In the present paper we apply this theory to the study of the phase diagram of the sys- 


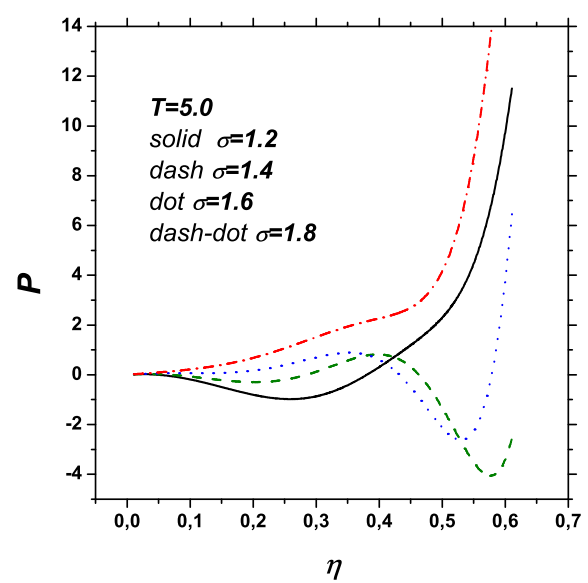

FIG. 2: The pressure isotherms for the system with $\varepsilon / \varepsilon_{1}=5$ for different values of $\sigma / d$ for $T=5\left(\sigma_{1}=2\right)$.

tem of particles interacting through an isotropic potential with an attractive well and a repulsive component consisting of a hard core plus a finite shoulder. This potential can be written in the form:

$$
\Phi(r)=\left\{\begin{array}{ll}
\infty, & r \leq d \\
\varepsilon_{1}, & d<r \leq \sigma \\
\varepsilon_{2}, & \sigma<r \leq \sigma_{1} \\
0, & r>\sigma_{1}
\end{array} .\right.
$$

The potential is shown in Fig. (11). We apply to the problem the first order thermodynamic perturbation theory for fluids. The soft core of the potential (11) is treated as perturbation with respect to the hard sphere potential. In this case the free energy of the system may be written in the form [29, 30]:

$$
\frac{F-F_{H S}}{N k_{B} T}=\frac{1}{2} \rho \beta \int u_{1}(r) g_{H S}(r) d \mathbf{r},
$$

where $\rho=V / N$ is the mean number density, $\beta=1 / k_{B} T$, $u_{1}(r)$ is the perturbation part of the potential $u_{1}(r)=$ $\Phi(r)-\Phi_{H S}(r), \Phi_{H S}(r)$ is the hard sphere singular potential, $g_{H S}(r)$ is the hard sphere radial distribution function, which is taken in the Percus-Yevick approximation 31.

To calculate $F_{H S}$, one can use, for example, the approximate equation [30]:

$$
\frac{F_{H S}}{k_{B} T N}=3 \ln \lambda-1+\ln \rho+\frac{4 \eta-3 \eta^{2}}{(1-\eta)^{2}} .
$$

Here $\lambda=h /\left(2 \pi m k_{B} T\right)^{1 / 2}$ and $\eta=\pi \rho \sigma^{3} / 6$.

Further in this paper we use the dimensionless quantities: $\tilde{\mathbf{r}}=\mathbf{r} / d, \tilde{P}=P \sigma^{3} / \varepsilon, \tilde{V}=V / N \sigma^{3}=1 / \tilde{\rho}$, $\tilde{T}=k_{B} T / \varepsilon$, omitting the tilde marks.

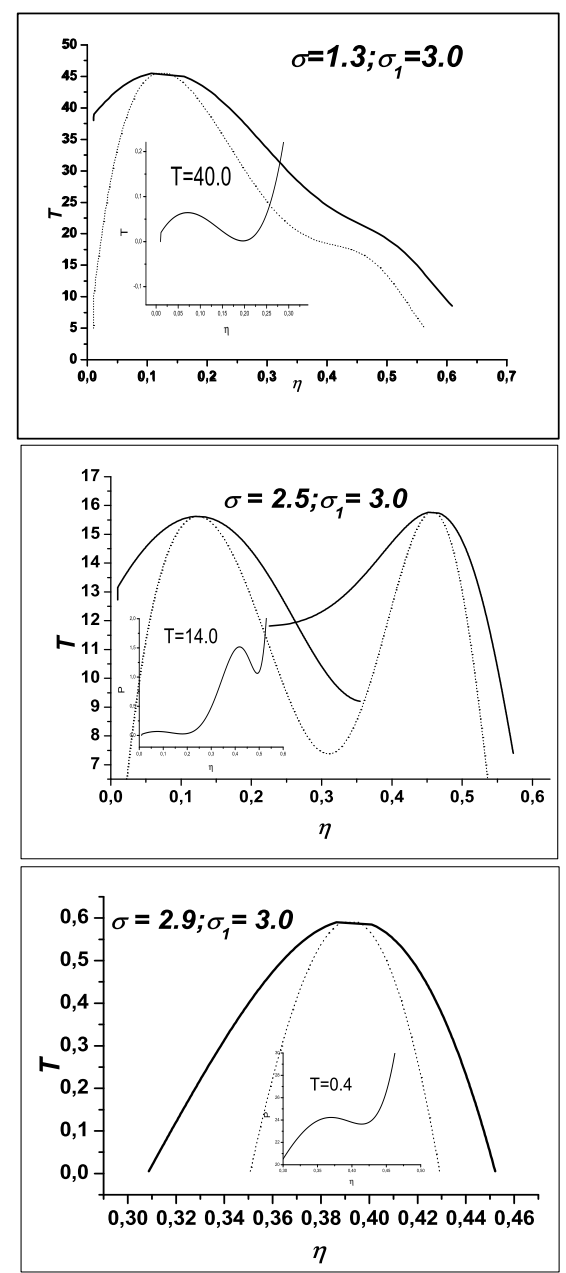

FIG. 3: Phase diagram of the system of particles interacting through the potential (1) for different values of $\sigma / d$ and fixed value of $\sigma_{1} / d=3$. Solid lines correspond to binodals and the dashed lines - to spinodals. Inserts show the characteristic isotherms.

Results of calculations are demonstrated in Figs. 24 In Fig. 2 a family of pressure isotherms is shown for the system with $\varepsilon / \varepsilon_{1}=5$ for different values of $\sigma / d$ for $T=5$. The van der Waals loops in the isotherms at low temperatures are clearly seen, this indicates the existence of the first order liquid-gas and liquid-liquid phase transitions.

Using the Maxwell construction we are able to calculate the equilibrium lines of the liquid-liquid phase transitions at different values of $\sigma / d$ (Figs. 3.4) (binodals) which are shown as solid lines in the figures. The dashed lines correspond to spinodals which as usual are calculated from the condition $\partial P / \partial \rho=0$. In Fig. 3] the evolution of the phase diagram is shown as a function of $\sigma / d$ for the fixed value of $\sigma_{1} / d=3$. We can see that the phase diagram starts from one gas-liquid transition for $\sigma / d=1.3$. If value of $\sigma / d$ increases the second (liquid- 

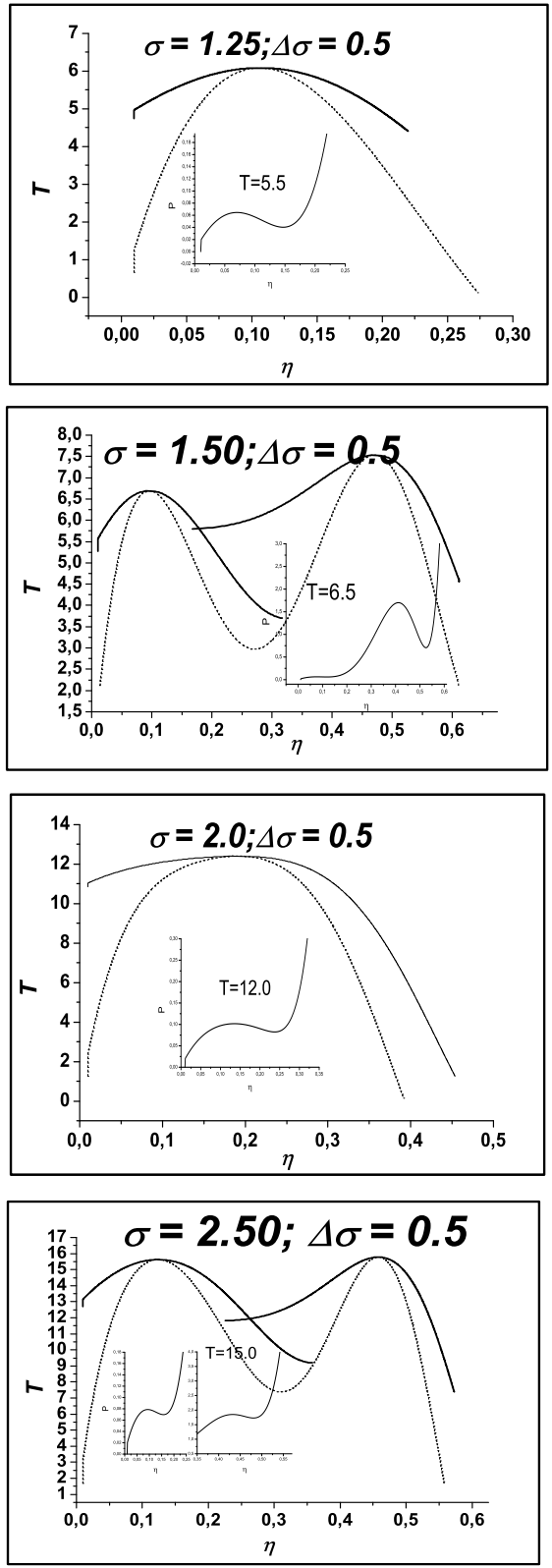

FIG. 4: Phase diagram of the system of particles interacting through the potential (1) for different values of $\sigma / d$ and fixed value of $\Delta \sigma=\sigma_{1} / d-\sigma / d=0.5$. Solid lines correspond to binodals and the dashed lines - to spinodals. Inserts show the characteristic isotherms.

liquid) transition develops. When difference $\sigma_{1} / d-\sigma / d$ is small enough $(\approx 0.2)$ the gas-liquid transition disappears, and one has the only liquid-liquid transition, as was discussed in Ref. 28]. We cannot extend the transition lines down to zero temperature and make calculations for $\sigma_{1} / d<1.3$ because of limitation of the pertur-

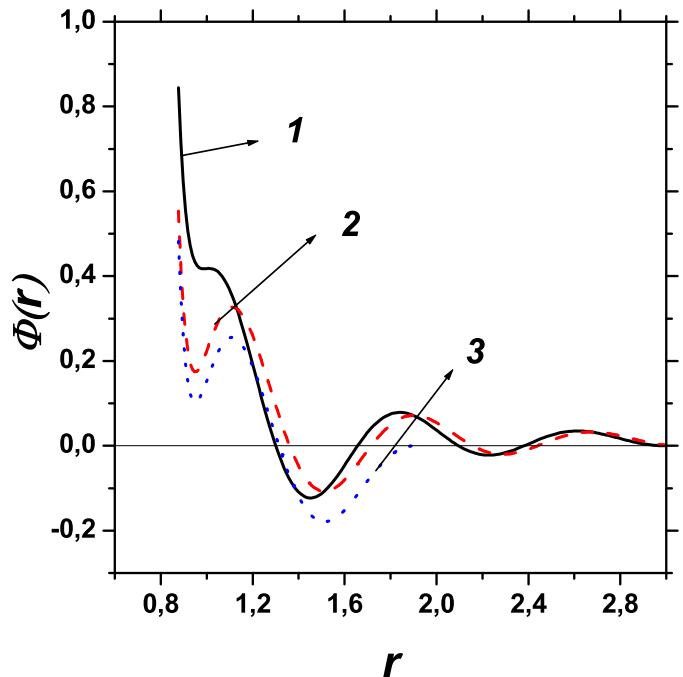

FIG. 5: The potential (4) for $r_{0}=1.1$ (line 1) and $r_{0}=1.16$ (line 2). The line 3 is obtained from the line 2 by the shift downward on the height of the first maximum.

bation approach.

Fig. [4 shows the evolution of the phase diagram of the system of particles interacting through the potential (1) for different values of $\sigma / d$ and fixed value of the difference $\Delta \sigma=\sigma_{1} / d-\sigma / d=0.5$. It should be noted that in contrast to Fig. 3 the liquid-liquid phase transition disappear for values of $\sigma$ in the vicinity of $\sigma \approx 2$. This seems to contradict the assertion in Refs. 24, 25 that the existence of a liquid-liquid phase transition is simply determined by the some kind of a balance between the repulsive and attractive parts of the potential, but depends also on the positions of the maxima of a reference radial distribution function (or the structure of the reference liquid). This is seen from the right hand side term of Eq. (11).

To illustrate the application of the generalized van der Waals theory to other systems let us consider the potential of the form:

$$
\Phi(r)=\frac{a \exp (-\alpha r) \cos \left(2 k_{f}\left(r-r_{0}\right)\right)}{r^{3}}+b\left(\frac{\sigma}{r}\right)^{18},
$$

where $\alpha=0.1, a=0.5, k_{f}=4.1, \sigma=0.331, b=$ $0.42 \times 10^{8}$. This potential may be used, for example, for a qualitative modelling of effective potentials of some metals 32. In Fig. [ this potential is shown for $r_{0}=1.1$ (line 1 ) and $r_{0}=1.16$ (line 2). The line 3 is obtained from the line 2 by the shift downward on the height of the first maximum.

In Fig. 6 the corresponding families of isotherms are shown for different temperatures. To calculate the equation of state corresponding to the potential (4) we apply the thermodynamic perturbation theory. The second 
part of the potential (4) was considered as a reference system.

$$
\Phi_{0}(r)=b\left(\frac{\sigma}{r}\right)^{18}
$$

Potential $\Phi_{0}(r)$ is approximated by the hard sphere potential with an effective diameter which depends on the density and the temperature 33].

From Fig. [6] one can see that there are only highdensity liquid-liquid transitions for the potentials corresponding to the lines (1)and (2) in Fig. 5 (upper figure). These transitions exist due to negative curvature of the potential (4). There are no gas-liquid transitions in these cases because the strength of an attraction is insufficient. For the potential corresponding to the line (3) in Fig. 5 there is also a low-temperature gas-liquid transition shown in the insert in the lower figure.

We would like to emphasize that we do not claim that the first order perturbation scheme, which was used in the present article, gives the high precision quantitative results, however, it seems reliable enough to give correct qualitative description of the liquid-liquid transition in the system with the potentials (11) and (4). It should be noted that the second-order perturbation theory gives qualitatively the same results.

Finally, let us make some remarks on another possible mechanism of a liquid-liquid phase transition. The crystalline solid state is characterized by a long-range positional order of the atomic density and associated bond orientational order belonging to one of the well-known allowed crystalline symmetries. In a diffraction experiment, $\delta$-function Bragg reflections are observed in the structure factor $S(\mathbf{q})$ that shows the associated point symmetry of the lattice. In the case of the long range bond-orientational order, one can observe the modulation of the structure factor which corresponds to the symmetry of the nearest neighbor environment of a particle (see, for example, 34]).

However, one can imagine the situation when the isotropy of three or four-particle correlation function is broken, but the symmetry of one and two-particle correlation functions is unchanged. In this case the structure factor of the system is the same as for the isotropic liquid because it depends only on the two-particle correlation function. This is why we call this type of the symmetry a "hidden symmetry" - it can not be detected in diffraction experiments.

So, using the diffraction experiments it is impossible to distinguish a liquid-liquid transition without changing the symmetry of the correlation functions and the transition accompanied by the breaking of a hidden symmetry. It seems that the only way to answer the question what kind of transition one observes in an experiment is to analyze the whole phase diagram. In principle the hidden symmetry transition may be of the first order, but in contrast to the liquid-liquid transition discussed in this article, it separates the phases with different symmetries and can not end at the critical point. There are two pos-
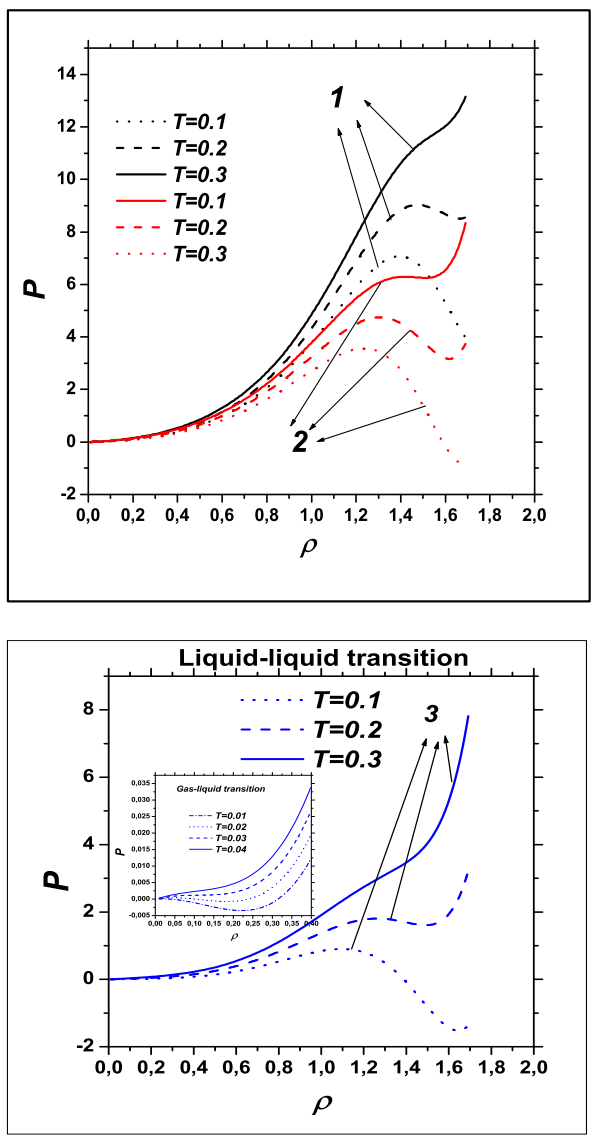

FIG. 6: The families of isotherms corresponding to the potentials in Fig. 5 There are only liquid-liquid transitions for the potentials corresponding to the lines (1)and (2) in Fig. 5 (upper figure). For the potential corresponding to the line (3) in Fig. 5] there is also a low-temperature gas-liquid transition shown in the insert in the lower figure.

sibilities for the hidden symmetry phase transition:(1) it may end at the tricritical point, and measurements above the tricritical point should reveal the thermodynamic anomalies but no density change; (2) it may intersect the gas-liquid transition line. In this case one can expect a sharp bend on the gas-liquid transition line at the point of intersection.

To our knowledge there are no calculations exploring the idea of breaking of the symmetry of higher order distribution functions in application to the problem of liquid-liquid transitions.

\section{Acknowledgments}

We thank S. M. Stishov, V. V. Brazhkin and A. G. Lyapin for stimulating discussions. The work was sup- 
ported in part by the Russian Foundation for Basic Research (Grants No 05-02-17280 and No 05-02-17621) and
NWO-RFBR Grant No 047.016.001.

Stanley, Phys. Rev. E 60, 6714 (1999).

[19] G. Franzese, G. Malescio, A. Skibinsky, S. V. Buldyrev and H. E. Stanley, Nature 409, 692 (2001).

[20] G. Franzese, G. Malescio, A. Skibinsky, S. V. Buldyrev and H. E. Stanley, Phys. Rev. E 66, 051206 (2002).

[21] E. A. Jagla, J. Chem. Phys. 111, 8980 (1999).

[22] G. Malescio and G. Pellicane, Phys. Rev. E 63, 020501(R) (2001).

[23] P. G. Debenedetti, Metastable liquids: Concepts and Principles (Princeton University Press, Princeton, NJ, 1998).

[24] A. Skibinsky, S. V. Buldyrev, G. Franzese, G. Malescio, and H. E. Stanley, Phys. Rev. E 69, 061209 (2004).

[25] G. Malescio, G. Franzese, A. Skibinsky, S. V. Buldyrev, and H. E. Stanley, Phys. Rev. E 71, 061504 (2005).

[26] E. A. Jagla, J. Chem. Phys. 111, 8980 (1999).

[27] E. A. Jagla, Phys. Rev. E 63, 061501 (2001).

[28] V. N. Ryzhov and S. M. Stishov, Phys. Rev. E 67, 010201(R) (2003).

[29] J.A. Barker and D. Henderson, J. Chem. Phys. 47, 2856 (1967).

[30] J.A. Barker and D. Henderson, Rev. Mod. Phys. 48, 587 (1976).

[31] W. R. Smith and W. Henderson, Mol. Phys. 19, 411 (1970).

[32] J.-F. Wax, R. Albaki, and J.-L. Bretonnet, Phys. Rev. B 62, 14818 (2000).

[33] L. Verlet and J.-J. Weis, Phys. Rev. A 5, 939 (1972).

[34] V.N. Ryzhov, J. Phys.: Condens. Matter 2, 5855(1990).

Stanley, Phys. Rev. Lett. 81, 4895 (1998).

[18] M. R. Sadr-Lahijany, A. Scala, S. V. Buldyrev and H. E. 